\title{
The ubiquitin ligase tripartite-motif-protein 32 is induced in Duchenne muscular dystrophy
}

\author{
Stefania Assereto ${ }^{1,6}$, Rosanna Piccirillo ${ }^{2,6}$, Serena Baratto ${ }^{1}$, Paolo Scudieri ${ }^{3}$, Chiara Fiorillo ${ }^{1}$, Manuela Massacesi ${ }^{1}$, \\ Monica Traverso ${ }^{1}$, Luis J Galietta ${ }^{3}$, Claudio Bruno ${ }^{4}$, Carlo Minetti ${ }^{1,5}$, Federico Zara ${ }^{1}$ and Elisabetta Gazzerro ${ }^{1}$
}

Activation of the proteasome pathway is one of the secondary processes of cell damage, which ultimately lead to muscle degeneration and necrosis in Duchenne muscular dystrophy (DMD). In $m d x$ mice, the proteasome inhibitor bortezomib up-regulates the membrane expression of members of the dystrophin complex and reduces the inflammatory reaction. However, chronic inhibition of the $26 \mathrm{~S}$ proteasome may be toxic, as indicated by the systemic side-effects caused by this drug. Therefore, we sought to determine the components of the ubiquitin-proteasome pathway that are specifically activated in human dystrophin-deficient muscles. The analysis of a cohort of patients with genetically determined DMD or Becker muscular dystrophy (BMD) unveiled a selective up-regulation of the ubiquitin ligase tripartite motif-containing protein 32 (TRIM32). The induction of TRIM32 was due to a transcriptional effect and it correlated with disease severity in BMD patients. In contrast, atrogin 1 and muscle RING-finger protein-1 (MuRF-1), which are strongly increased in distinct types of muscular atrophy, were not affected by the DMD dystrophic process. Knock-out models showed that TRIM32 is involved in ubiquitination of muscle cytoskeletal proteins as well as of protein inhibitor of activated STAT protein gamma (Piasy) and N-myc downstream-regulated gene, two inhibitors of satellite cell proliferation and differentiation. Accordingly, we showed that in DMD/BMD muscle tissue, TRIM32 induction was more pronounced in regenerating myofibers rather than in necrotic muscle cells, thus pointing out a role of this protein in the regulation of human myoblast cell fate. This finding highlights TRIM32 as a possible therapeutic target to favor skeletal muscle regeneration in DMD patients.

Laboratory Investigation (2016) 96, 862-871; doi:10.1038/labinvest.2016.63; published online 13 June 2016

Duchenne muscular dystrophy (DMD) is a recessive $\mathrm{X}$-linked disorder affecting about one of every 3500 males, and it involves progressive muscle degeneration and weakness, causing premature death. The disease results from mutations of the $D M D$ gene, which lead to deficiency of dystrophin, a 427 kiloDalton $(\mathrm{kDa})$ protein found throughout the cytoplasmic face of the plasma membrane in both skeletal and cardiac muscles. Becker muscular dystrophy (BMD), a milder allelic form of the disease, is caused by a reduction in the amount of dystrophin or a change in its size. ${ }^{1,2}$

Dystrophin is an important structural component of skeletal muscle: it provides stability to the dystrophinglycoprotein complex (DGC) on the cell membrane and assures a link between the cytoskeleton and the extracellular matrix. Dystrophin deficiency is associated with a decrease in or even loss of the expression and membrane localization of members of DGC, such as sarcoglycans, dystroglycans, and syntrophins. Plasma membrane instability caused by loss of mechanical binding with cytoskeletal F-actin leads to disarray of the multimeric complex and diverts its components to proteolysis. $^{3}$

Misfolding, aggregation, and tissue accumulation of undesired proteins are common events underlying different diseases, and cells have developed complex and complementary pathways aimed at preserving protein homeostasis. These protective pathways include the unfolded protein response, the ubiquitin-proteasome system, the autophagic-lysosomal pathway, and the encapsulation of damaged proteins in aggresomes. ${ }^{4}$

In skeletal muscle, the ubiquitin-proteasome system has an important role in the maintenance of muscle mass and proteasome dysfunctions are now linked to genetic primary myopathies. ${ }^{5-7}$

\footnotetext{
'Unit of Paediatric Neurology and Muscle Diseases, G. Gaslini Institute, Genoa, Italy; ${ }^{2}$ Department of Oncology, IRCCS-Mario Negri Institute for Pharmacological Research, Milano, Italy; ${ }^{3}$ Unit of Medical Genetics, G. Gaslini Institute, University of Genoa, Genoa, Italy; ${ }^{4}$ Department of Neuroscience, Center of Myology and Neurodegenerative Disorders, G. Gaslini Institute, Genoa, Italy and ${ }^{5}$ Department of Neuroscience, and Pediatrics (DINOGMI), University of Genoa, Genoa, Italy.

Correspondence: Dr E Gazzerro MD, Unit of Paediatric Neurology and Muscle Diseases, G. Gaslini Institute, L.go Gaslini 5, Genova 16147, Italy.

E-mail: elisabettagazzerro@ospedale-gaslini.ge.it

${ }^{6}$ These authors contributed equally to this work.
}

Received 15 December 2015; revised 19 April 2016; accepted 25 April 2016 
We previously showed that in $m d x$ mice, a naturally occurring mouse model of DMD, systemic treatment with the proteasome inhibitors (PIs) MG-132 or bortezomib (Velcade) up-regulated the membrane expression of DGC members and reduced the inflammatory reaction related to myofiber damage, thus improving the dystrophic phenotype. ${ }^{3,8,9}$ Similarly, in golden retriever muscular dystrophy, bortezomib improved a few histopathological features of dystrophindeficient skeletal muscles and blocked the activation of the pro-inflammatory pathway triggered by phospho-nuclear factor $\mathrm{kB} .{ }^{10,11}$

However, although bortezomib is used for the treatment of human relapsing multiple myeloma and mantle cell lymphoma, its elective application in the clinical setting is limited because of various troublesome side-effects (painful neuropathy in $>30 \%$ of patients, thrombocytopenia, hypotension, fatigue). ${ }^{12-14}$ Moreover, chronic generalized inhibition of the $20 \mathrm{~S}$ subunit of the proteasome may be ineffective or even potentially deleterious, possibly because of undesirable accumulation of mutated proteins and unsustainable perturbation of cellular protein homeostasis (proteostasis). ${ }^{5,15,16}$

Indeed, in skeletal muscle, proteasome activation is also involved in physiological processes such as muscle development, in which dramatic changes in protein expression and cell morphology rely on the turnover of regulatory and structural components. ${ }^{17}$

Therefore, to identify selective therapeutic targets, it is essential to dissect the molecular cascade, which leads to the degradation of DGC proteins through the ubiquitinproteasome system pathway in dystrophin-deficient skeletal muscle.

In eukaryotic cells, the specificity of intracellular ubiquitinproteasome system protein degradation is achieved mainly by a specific substrate-marking system involving the heat-shock protein, ubiquitin $(\mathrm{Ub})$. Protein ubiquitination is the result of sequential reactions catalyzed by three types of enzymes namely E1 (Ub-activating enzyme, 2 isoforms per cell), E2 (Ub-carrier protein, 20-40 isoforms per cell), and E3 (Ubligase protein, 500-1000 isoforms per cell), which recognize and bind target proteins, conferring substrate selectivity to this process. Ubiquitinated proteins are then rapidly recognized and cut into small peptides by the $26 \mathrm{~S}$ proteasome. ${ }^{18}$

Muscle RING-finger 1 (MuRF-1) and muscle atrophy F-box (MAFbx)/atrogin1 were identified more than 10 years ago as two Ub ligases induced during muscle atrophy. ${ }^{18,19}$ However, C terminus of Hsc70-interacting protein ( $\mathrm{CHiP})$, tripartite motif-containing protein 32 (TRIM32), neural precursor cell expressed developmentally down-regulated protein 4 (NEDD4) and neuralized-like protein 2 (Ozz) have also been shown to exert distinct physiological activities in skeletal muscle. ${ }^{20-23}$

The purpose of this study was to evaluate the expression of these Ub ligases/E3s in human dystrophin-deficient muscle tissues, as their selective modulation could represent a novel therapeutic strategy for ubiquitin-proteasome system regulation in DMD/BMD.

\section{MATERIALS AND METHODS Muscle Biopsies}

All human samples were collected after patients had signed informed consent forms in accordance with the requirements of the G. Gaslini Institute Ethics Committee. Tissue sections were isolated from muscle biopsies on the femoral quadriceps obtained for diagnostic purposes from six patients with DMD, eight patients with BMD, and six healthy individuals. The diagnosis of DMD or BMD was genetically confirmed.

Morphological score of BMD disease severity was established through standard hematoxylin \& eosin (H\&E) staining of tissue sections. The presence and degree of degeneration/ necrosis, fibrotic replacement, nuclear centralization, and hypercontracted muscle fibers were blindly quantified according to arbitrary criteria by two independent morphologists.

\section{Proteasome Activity}

The $26 \mathrm{~S}$ chymotryptic proteasome activity was determined by the cleavage of specific fluorogenic substrates in skeletal muscle tissue from six DMD patients and four healthy controls. Briefly, the tissue was homogenized in $20 \mathrm{mmol} / \mathrm{l}$ Tris- $\mathrm{HCl}, \mathrm{pH} 7.2$, containing $0.1 \mathrm{mmol} / \mathrm{l}$ EDTA, $1 \mathrm{mmol} / \mathrm{l}$ 2-mercaptoethanol, $5 \mathrm{mmol} / \mathrm{l}$ ATP, $20 \%$ glycerol, and $0.04 \%$ (v/v) Nonidet P- 40 . Muscle homogenates were then centrifuged at $13000 \mathrm{~g}$ for $15 \mathrm{~min}$ at $4{ }^{\circ} \mathrm{C}$. The supernatant was collected and the protein concentration determined. Aliquots of $100 \mu \mathrm{g}$ protein were then incubated for $60 \mathrm{~min}$ at $37^{\circ} \mathrm{C}$ with a specific fluorogenic substrate (Suc-Leu-Leu-Val-Tyr-7-AMC; Sigma-Aldrich, Milan, Italy) in $50 \mathrm{mmol} / \mathrm{l} \mathrm{Hepes,} \mathrm{pH}$ 8.0, $5 \mathrm{mmol} / \mathrm{l}$ EGTA buffer. Fluorescence was assessed with spectrofluorometer $(380 \mathrm{~nm}$ excitation, $460 \mathrm{~nm}$ emissions; Perkin-Elmer, Norwalk, CT, USA). The activity, obtained by measuring the release of free AMC, is expressed as relative fluorescence units and reported as medians and first/third quartiles (Q1, Q3); $P<0.05$ was considered significant.

\section{RNA and Protein Extraction}

Total RNA and total proteins were extracted from skeletal muscle tissue samples (from 20 to $55 \mathrm{mg}$ ) of six healthy controls, six DMD, and eight BMD patients using the RNeasy Lipid Tissue Mini Kit (Qiagen, Milan, Italy) according to the manufacturer's protocol including DNase treatment. Quality and quantity of RNA were analyzed using a Thermo Scientific NanoDrop 1000 Spectrophotometer.

Total protein fractions were isolated from the organic phase of QIAzol Reagent-treated samples during the lipid extraction of RNA. This protocol requires a protein precipitation step with isopropanol and a series of purifications with guanidine-ethanol solution. The pellet containing proteins was finally resuspended with $500 \mu$ l of UREA/DTT solution. Protein concentration was determined by the Bradford protein assay. 
Isolation of Peripheral Blood Mononuclear Cells (PBMCs) Mononucleated cells were purified from $5 \mathrm{ml}$ of venous peripheral blood from three healthy controls, three patients affected by BMD, and two patients affected by DMD after obtaining their informed consent. Cells were isolated by density gradient centrifugation with Lympholyte- $\mathrm{H}$ separation medium (Cedarlane, Burlington, Canada) following manufacturer's instructions.

Total RNA from PBMCs was extracted using the RNeasy Mini Kit (Qiagen) according to the manufacturer's protocol including DNase treatment. Quality and quantity of RNA were determined using a NanoDrop spectrophotometer. The cDNA was synthesized from $400 \mathrm{ng}$ of total RNA with the iScript cDNA Synthesis Kit (Bio-Rad). Each RNA sample was checked for genomic DNA contamination.

\section{Quantitative PCR}

Quantitative real-time PCR (qRT-PCR) was carried out on RNA extracted from skeletal muscle tissue of patients or controls. The cDNA was synthesized from $350 \mathrm{ng}$ of total RNA with the iScript cDNA Synthesis Kit (Bio-Rad Laboratories, Milan, Italy). Each RNA sample was checked for genomic DNA contamination without reverse transcriptase addition into cDNA synthesis mixture. qRT-PCR was run in triplicate with the $2 \times$ Sso Fast EvaGreen Supermix (BioRad) in a CFX96 Real-time PCR detection system (Bio-Rad). The $15 \mu \mathrm{l}$ PCR mixture contained diluted cDNA corresponding to $8.75 \mathrm{ng}$ of total RNA and $0.25 \mu \mathrm{M}$ of each primer. TRIM32 relative expression levels were normalized to a calibrator sample by using the comparative $\mathrm{Ct}(\Delta \Delta \mathrm{Ct})$ method, and to the geometric average of a set of two housekeeping genes by the Bio-Rad CFX manager software, version 3.1..$^{24,25}$ For each specific primer set, the efficiency was $>95 \%$ and a single product was seen on the melting curve analysis. Specific primers for human TRIM32 (NM_012210), human myosin-heavy chain, MYH1 (NM_005963), human actin, alpha 1, skeletal muscle, ACTA1 (NM_001100) were designed with Beacon Designer 2.0 Software. TRIM32-F 5'-GG CTCTGTAGGCCCTGATG-3', TRIM32-R 5'-TGCAGCGGAA ATCCTCATTC-3'; MYH-F 5'-GAAGGAAGGCATTGAGTG-3', MYH-R 5'-TTGAAGGAGGTGTCTGTC-3'; ACTA-F 5'-TATC GAGCACGGCATCATCA-3', ACTA-R 5'-TAAATGGGCACGT TGTGGGT-3'.

\section{Western Blot Analysis}

Equal amounts of proteins $(80 \mu \mathrm{g})$ mixed with $4 \times$-sodium dodecyl sulfate (SDS) gel-loading buffer $(100 \mathrm{mM}$ Tris-HCl pH 6.8, $200 \mathrm{mM}$ dithiothreitol, 4\% SDS, $0.2 \%$ bromophenol blue, $20 \%$ glycerol) and denatured at $95^{\circ} \mathrm{C}$ for $5 \mathrm{~min}$, were loaded into each lane and resolved in 12\% SDSpolyacrylamide gel.

The proteins were blotted on PVDF membranes (Immobilon PVDF, Millipore), which were then blocked in $5 \%$ bovine serum albumin (BSA) in PBS Tween 0.05\% (PBSt). Membranes were probed with primary antibodies to MuRF-1 (molecular weight (MW) $40 \mathrm{kDa}$, see below), Atrogin 1 (MW $25 \mathrm{kDa}$, a kind gift of Dr S. Lecker from Beth Israel Deaconess Medical Center in Boston, USA), Neural precursor cell expressed developmentally downregulated protein 4 (NEDD4) (MW $104 \mathrm{kDa}$, Abcam, Cambridge, UK), Tripartite motif-containing protein 32 (TRIM32) (MW $72 \mathrm{kDa}$, Novus Biological, Littleton, CO USA), $\mathrm{C}$ terminus of HSC70-interacting protein ( $\mathrm{CHiP}$ ) (MW $35 \mathrm{kDa}$, Calbiochem, Milan, Italy), Neuralized-like protein 2 (Ozz) (MW $29 \mathrm{kDa}$, Santa Cruz Biotech Heidelberg, Germany), $20 \mathrm{~S}$ proteasome core subunits rabbit (MW 25-30kDa, Calbiochem, Milan, Italy).

Secondary antibodies were conjugated to alkaline phosphatase (Promega, Milan, Italy). Band intensities were analyzed using ImageJ software, version 1.45 (National Institutes of Health). The signals were developed using CDP-Star substrate (Life Technology, Monza, Italy). An antibody anti-GAPDH (MW $37 \mathrm{kDa}$, Sigma, Milan, Italy) was used as a loading

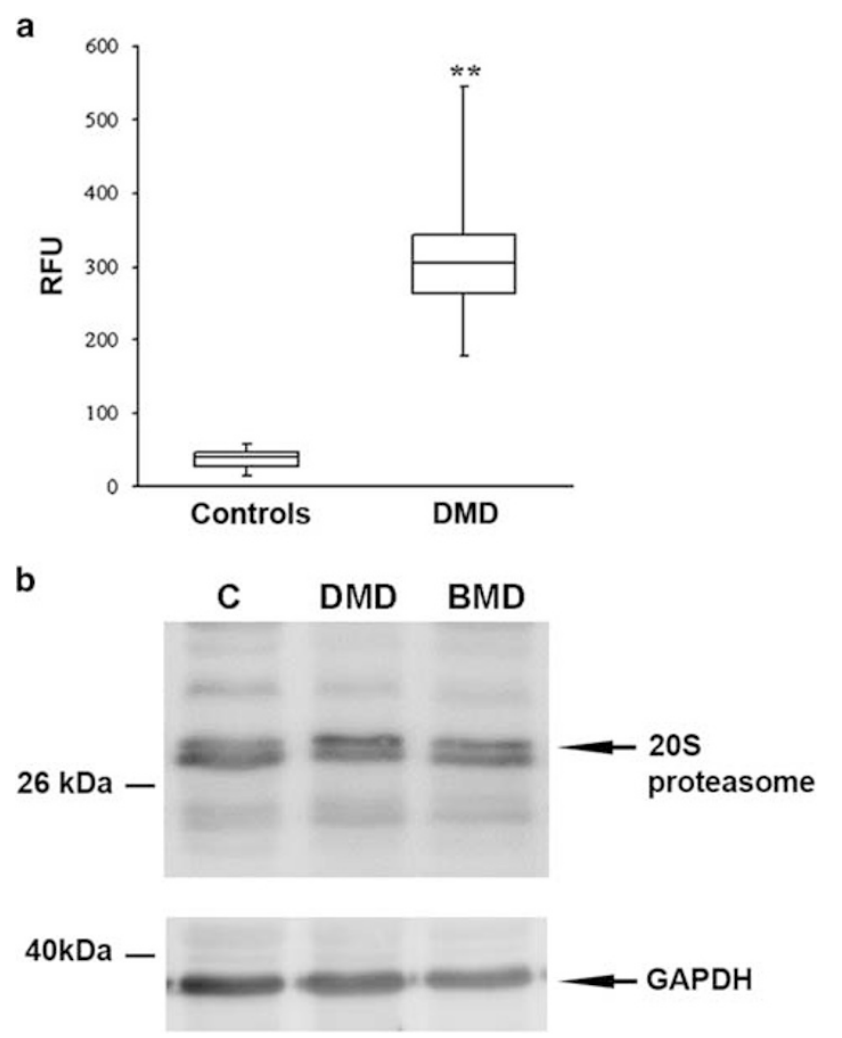

Figure 1 The 26S proteasome activity was significantly increased in DMD muscle lysates when compared to healthy controls. (a) Proteasome activity was evaluated in total lysates from the muscle biopsies of six DMD and four healthy controls by means of specific fluorogenic substrates. Each column represents the median \pm Q1, Q3 of relative fluorescence units; ${ }^{* *} P<0.01$ controls vs DMD. (b) Muscle lysates from four controls (C), four BMD and four DMD patients were separated by SDS-PAGE, and subjected to immunoblot analysis with a primary anti-20 S proteasome core subunits antibody. GAPDH was utilized as a loading control $(37 \mathrm{kDa})$. Representative images are shown. 
control. The MuRF-1 antibody was raised against rat MuRF-1, as previously described. ${ }^{18,19}$

\section{Immunofluorescence Staining}

Unfixed 5- $\mu \mathrm{m}$-thick cryosections of skeletal muscle biopsies from six DMD patients, eight BMD patients, and six agematched healthy controls were incubated in Da Vinci Green Diluent (BioCare Medical, Concord, CA, USA) for $10 \mathrm{~min}$ at room temperature (RT). Sections were incubated with 1:75 dilution of a monoclonal antibody anti-human TRIM32 (Abnova Corporation, Taipei, Taiwan), 1:20 dilution of a monoclonal antibody anti-human developmental type myosin-heavy chain antibody (Novocastra, Newcastle upon Tyne, UK), 1:20 dilution of a monoclonal antibody anti C5b9 (Dako, Glostrup, Denmark) overnight at RT, washed three times with phosphate buffered saline (PBS), and incubated with 1:750 dilution of secondary antibodies AlexaFluor antimouse $\operatorname{IgG}_{1}-488$ and AlexaFluor anti-mouse $\mathrm{IgG}_{2}-546$ (Invitrogen, Camarillo, CA, USA) for $1 \mathrm{~h}$ at RT. Sections were washed three times with PBS, slides were mounted with Vectashield Mounting Medium (Vector Laboratories,
Burlingame, CA, USA) and examined under a TCS SP8 confocal microscope (Leica).

\section{Statistical Analysis}

The distribution of analyzed variables was confirmed by the Shapiro-Wilk test. For parametric measures, values were expressed as means \pm standard deviations, and differences were evaluated by Student's $T$ test. For non-parametric variables, data were expressed as medians with first and third quartiles (Q) and differences were analyzed by nonparametric Mann-Whitney $U$ test. A $P$ value $<0.05$ was considered as statistically significant.

\section{RESULTS}

TRIM32 Protein Content is Increased in Femoral Quadriceps of DMD Patients

Proteasome activities (chymotrypisn-, trypsin-, and caspaselike) are overall increased in the hindlimb muscles isolated from the two available DMD mouse models, the $m d x$ and the double utrophin $/ m d x$ knock-out mice. ${ }^{6,8,9}$ To confirm the relevance of this observation in human DMD, we determined via a standard proteasome activity assay that the a

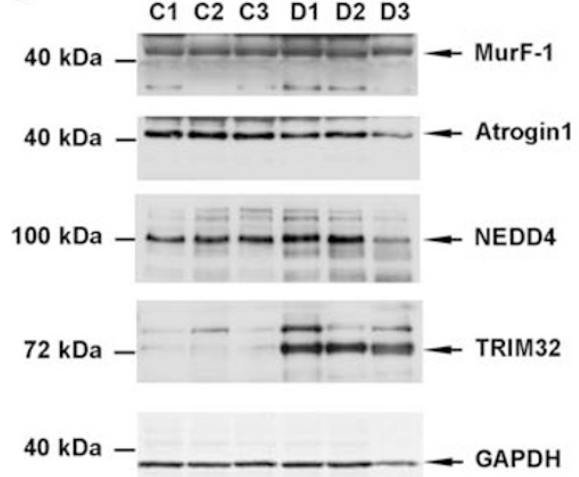

$\begin{array}{lllllll}C & C 2 & C & D & D & D 2 & D\end{array}$

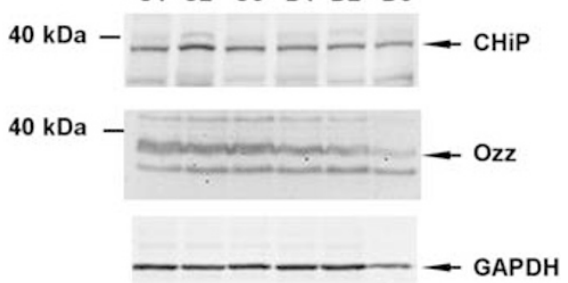

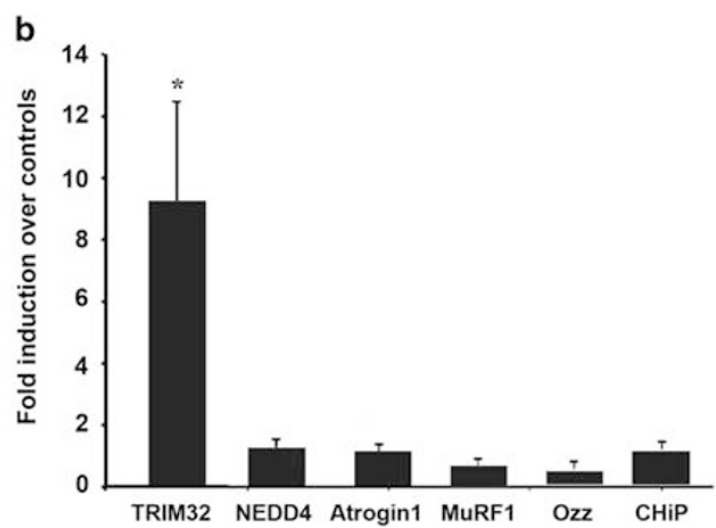

Figure 2 TRIM32 protein was induced in DMD muscle tissue. (a) Muscle lysates from six controls (C), and six DMD patients (D) were separated by SDS-PAGE, and subjected to immunoblot analysis with primary antibodies directed against MuRF-1 (40 kDa), Atrogin1 (40 kDa), NEDD4 (104 kDa), TRIM32 (72 kDa), CHiP (35 kDa), and Ozz (29 kDa). GAPDH was utilized as a loading control (37 kDa). Representative images are shown. (b) Bands quantitation was performed by densitometric analysis. Data (mean \pm standard deviations from six controls and six DMD patients) are expressed as fold induction of selected proteins in DMD patients vs controls. ${ }^{*} P<0.05$ controls vs DMD. 
chymotrypsin-like $26 \mathrm{~S}$ proteasome activity was six times higher in the muscle biopsies of six genetically determined DMD patients than in four healthy controls (Figure 1).

We next quantitated the protein levels of the Ub ligases Atrogin1, CHiP, MuRF-1, NEDD4, Ozz, and TRIM32 in muscle lysates of six DMD patients. TRIM32 resulted to be increased by approximately ninefold, whereas the other E3s were not affected (Figures $2 \mathrm{a}$ and $\mathrm{b}$ ).

TRIM32 induction was further confirmed by immunofluorescence studies: in control sections of six healthy individuals, TRIM32 was characterized by an extremely weak cytoplasmic staining, whereas in DMD patients it displayed an intense signal, particularly pronounced in organized clusters of muscle fibers and it showed a more specific subsarcolemmal localization (Figure 3). When all myofibers were blindly counted in the whole section, $\sim 12.5 \%(11.1-20)$, (median (Q1-Q3)) showed an intense TRIM32 signal (Figures 3a and b; Table 1).

TRIM32 up-regulation was partially due to a transcriptional effect since TRIM32 transcript levels were increased by
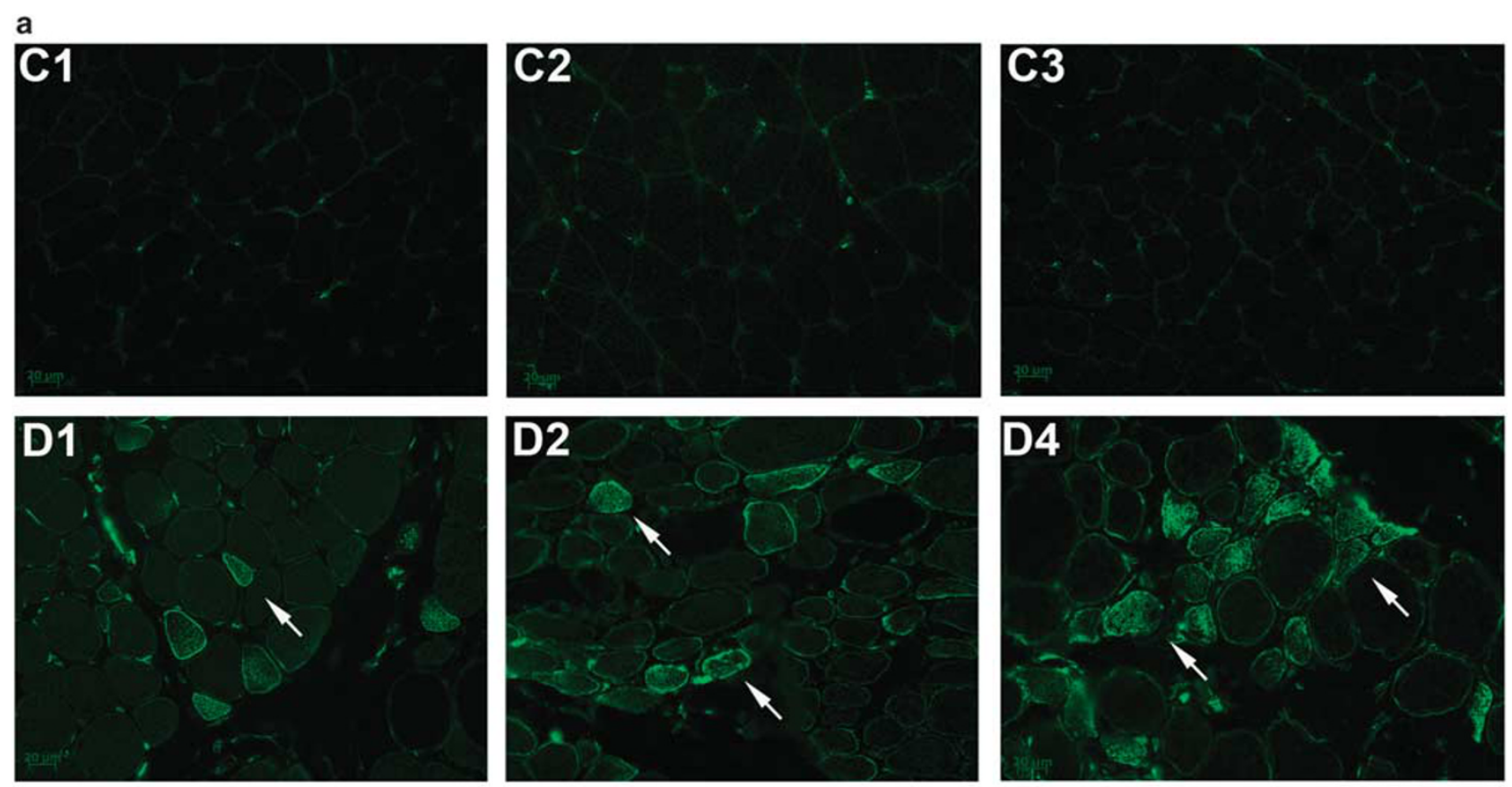

b
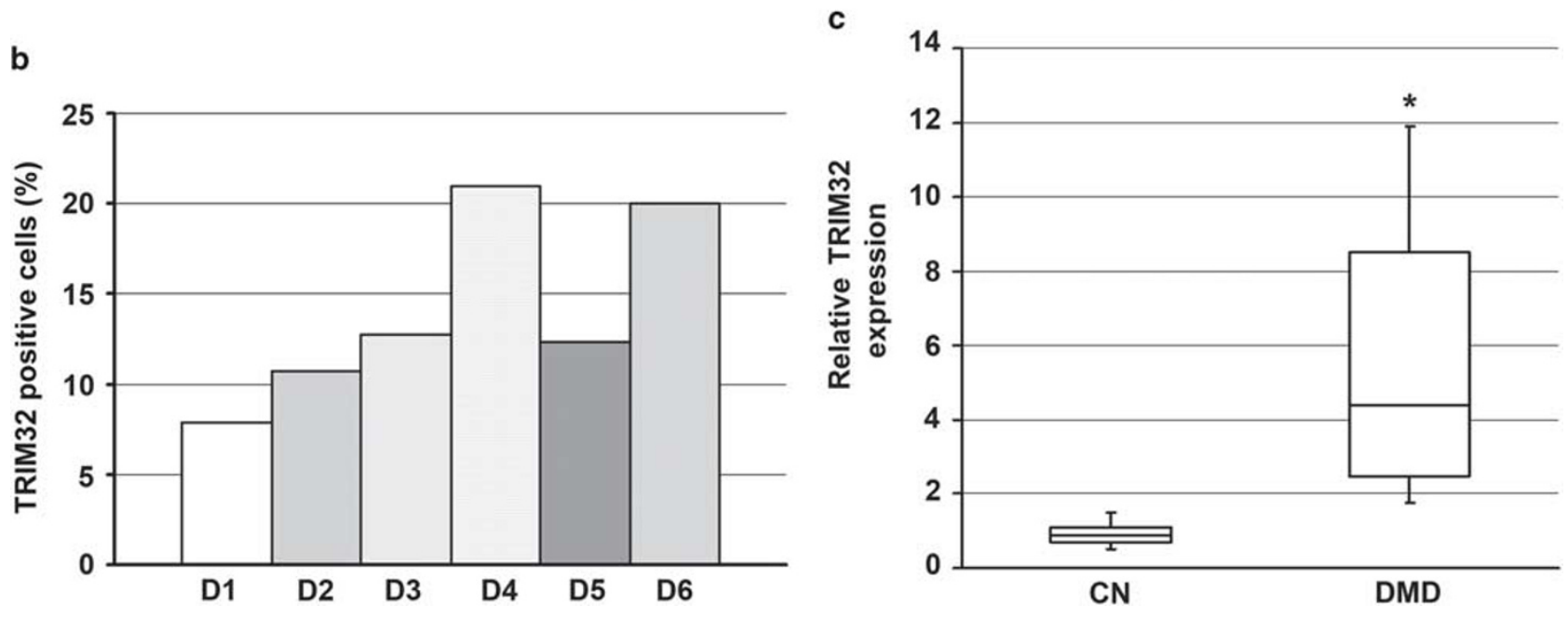

Figure 3 TRIM32 protein and transcripts were increased in DMD muscle tissue. (a) Frozen skeletal muscle sections from controls (C) and DMD patients (D) were immunostained with a monoclonal antibody directed against TRIM32 (white arrows). In sections from DMD patients, the protein displays a cytoplasmic and subsarcolemmal intense staining. Original magnification, $\times 40$. Representative images are shown. Scale bars $=20 \mu$ m. $(\mathbf{b})$ The number of TRIM32 ${ }^{+}$myofibers was blindly counted in all fields of DMD sections. (c) The analysis of TRIM32 transcript levels was performed with mix Sso Fast Evagreen on RNA extracted from the muscle biopsy of five controls and six DMD patients. The levels of expression of TRIM32 were normalized with two housekeeping genes: MYH1, adult skeletal muscle myosin-heavy chain 1; ACTA1, skeletal muscle actin, alpha 1. Each column represents the median \pm Q1, Q3; *P $<0.05$ controls vs DMD. 
Table 1 Histopathological features of DMD (D1 $\rightarrow$ D6) patients and number of TRIM32 positive fibers

\begin{tabular}{|c|c|c|c|c|c|c|c|c|}
\hline Patients & Mutation & Fibrosis & Necrosis & Degeneration & Centralized nuclei & Hypercontr. fibers & TRIM32+ fibers (\%) & Total score of severity \\
\hline D1 & Del 32-43 & $1-2$ & $2-3$ & $2-3$ & $1-2$ & 2 & 7.87 & 10 \\
\hline D2 & Del 8-9 & $2-3$ & $2-3$ & $1-2$ & 2 & $1-2$ & 12.30 & 10 \\
\hline D3 & Del 45 & $1-2$ & $2-3$ & $2-3$ & 2 & $1-2$ & 12.75 & 10 \\
\hline D4 & Del 44 & 2 & $2-3$ & $2-3$ & $1-2$ & 2 & 20.94 & 10.5 \\
\hline D5 & Del 45-52 & 2 & $2-3$ & $2-3$ & 2 & $2-3$ & 10.70 & 11.5 \\
\hline D6 & Del 49-52 & 2 & 2 & 2 & 2 & $2-3$ & 19.98 & 10.5 \\
\hline
\end{tabular}

Abbreviations: Del, deletion; Hypercontr., hypercontracted fibers.

Table 2 Histopathological features of BMD $(B 1 \rightarrow B 8)$ patients and number of TRIM32 positive fibers

\begin{tabular}{|c|c|c|c|c|c|c|c|c|}
\hline Patients & Mutation & Fibrosis & Necrosis & Degeneration & Centralized nuclei & Hypercontr. fibers & TRIM32+ fibers (\%) & Total score of severity \\
\hline B1 & Del 48-55 & $0-1$ & 0 & 0 & 0 & 0 & 0 & 0.5 \\
\hline B2 & Del 48-51 & 0 & 0 & 0 & 1 & 0 & 0 & 1 \\
\hline B3 & Del 48-55 & 1 & $0-1$ & $1-2$ & 1 & 1 & 0.78 & 5 \\
\hline B4 & Del 42-51 & 1 & 0 & 1 & 1 & 1 & 1.26 & 4 \\
\hline B5 & Del 48 & 1 & 0 & 0 & 1 & 3 & 2.78 & 5 \\
\hline B6 & Del 45-51 & 1 & $0-1$ & 1 & 1 & 3 & 3.74 & 6.5 \\
\hline B7 & Del 45-48 & -2 & $2-3$ & $2-3$ & $0-1$ & 3 & 11.7 & 10 \\
\hline B8 & Del 45-49 & $2-3$ & $2-3$ & $2-3$ & 2 & 3 & 19.9 & 12.5 \\
\hline
\end{tabular}

Abbreviations: Del, deletion; Hypercontr., hypercontracted fibers.

fivefold in muscles isolated from six DMD patients compared with five healthy controls (Figure 3c). Finally, the induction of TRIM32 transcripts seemed tissue-specific as it was not observed by qPCR in peripheral mononuclear cells isolated from DMD patients and healthy controls (data not shown).

\section{TRIM32 Protein Content is Increased in Regenerating Fibers}

To correlate TRIM32 induction with different stages of dystrophinopathy, TRIM32 levels were analyzed in skeletal muscle tissue samples isolated from BMD patients (Table 2).

BMD is a milder form of dystrophinopathy and is caused by in-frame mutations of the DMD gene. BMD is characterized by a wide phenotype range, and patients may display much milder weakness, with onset in middle or late adulthood and/or limited to the quadriceps muscles. ${ }^{1}$

Western blot analysis of eight BMD patients displaying muscular phenotypes with various degrees of severity showed that TRIM32 up-regulation was almost undetectable in the mildest BMD case (B1), whereas it was pronounced in the most severe one, being almost equivalent to a DMD patient (B8) (Figure 4c).
In immunohistochemistry, BMD sections with a highly compromised morphology displayed up to $20 \%$ TRIM32 positive fibers as compared with normal controls, which were substantially negative (Figures $4 \mathrm{a}$ and b). Intriguingly, as observed in DMD, most TRIM32 positive cells were grouped in clusters of small myofibers with central vacuolated nucleii, which are usually observed in regenerating myoblasts.

Consistently, TRIM32 signal co-localized with the regeneration marker developmental type myosin-heavy chain (MHCd, Figure 5), whereas it was not detected in necrotic myofibers positive for the degeneration marker C5b-9 (data not shown).

\section{DISCUSSION}

Recent evidence indicates that pharmacological treatments interfering with the ubiquitin-proteasome pathway in experimental in vitro and in vivo models of muscular dystrophies, such as sarcoglycanopathies, dysferlinopathies, and merosin-deficiencies, might prevent the removal of mutated proteins, promote functional recovery and improve the dystrophic phenotype associated with these disorders. ${ }^{26-33}$

However, degradation of proteins by the ubiquitinproteasome is a complex ATP-dependent multistage process 
a
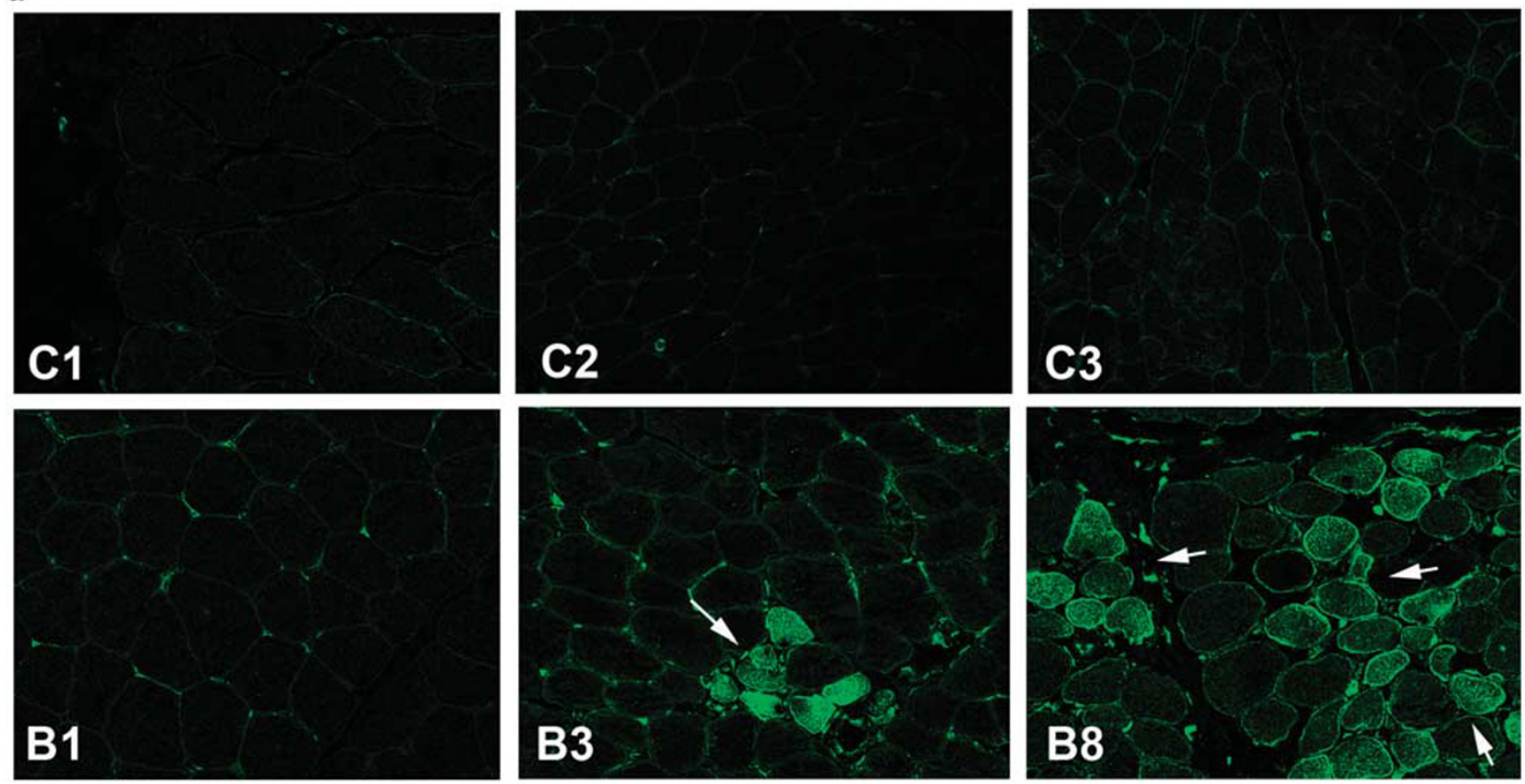

b
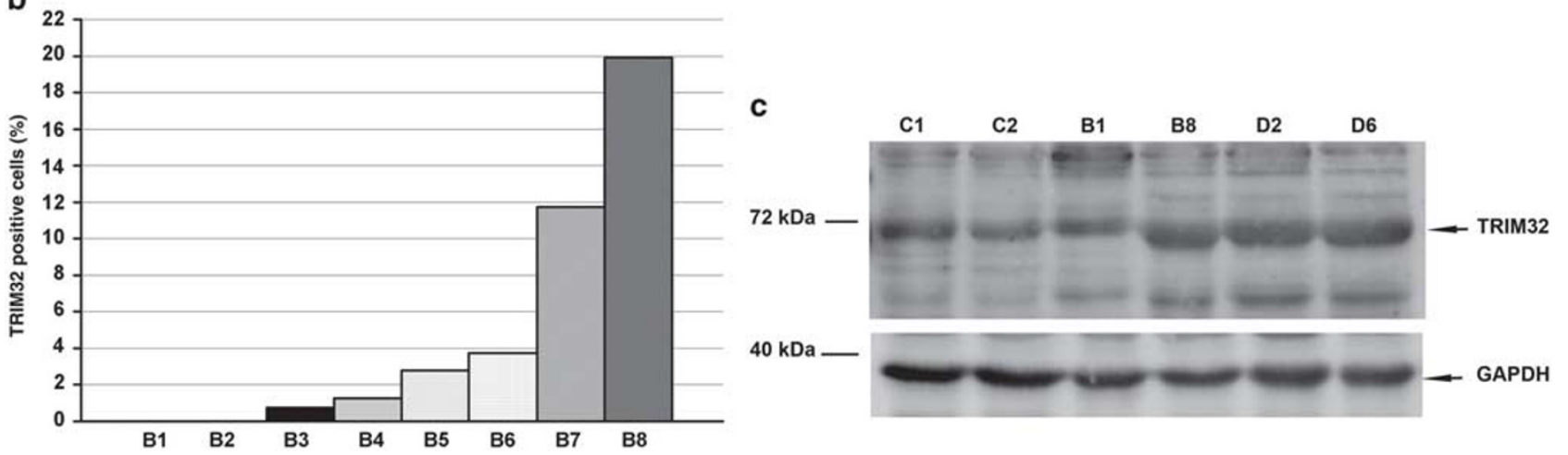

Figure 4 TRIM32 protein was significantly up-regulated in BMD muscle tissue. (a) Frozen skeletal muscle sections from three controls (C) and eight BMD patients (B) were immunostained with a monoclonal antibody directed against TRIM32. TRIM32 staining was significantly up-regulated in the BMD sections with a highly compromised morphology (white arrows). In these samples, the protein displays a cytoplasmic and subsarcolemmal intense staining. Original magnification, $\times 40$. Representative images are shown. Scale bars $=20 \mu \mathrm{m}$. (b) The number of TRIM32 $2^{+}$myofibers was blindly counted in all fields of BMD sections. (c) Muscle lysates from two controls (C), two BMD patients (B1 and B8) displaying a different degree of muscle damage according to the morphological score established by two independent morphologists (Table 2) and two DMD patients (D) were separated by SDS-PAGE and subjected to immunoblot analysis with primary antibody directed against TRIM32 (72 kDa). The data show that TRIM32 was increased in B8, i.e. the BMD patient with the most severe phenotype, and in DMD samples when compared to controls. GAPDH was utilized as a loading control ( $37 \mathrm{kDa}$ ).

that requires ubiquitination of the target protein by selective Ub ligases before its degradation. The exact molecular players involved in the activation of the ubiquitin-proteasome in the different muscular disorders are only partially known. ${ }^{34}$

Here, we showed that human skeletal muscle affected by dystrophin deficiency displays a selective induction of the Ub-ligase TRIM32. In contrast, atrogin1 (also known as F-box only protein 32, FBXO32) and MuRF-1 (TRIM63), the two Ub ligases strongly induced in different types of muscular atrophy, ${ }^{19}$ were not affected by the DMD dystrophic process.
TRIM32 is member of the tripartite-motif (TRIM) family of proteins. ${ }^{21,35}$ The TRIM motif includes three zinc-binding domains, a RING-finger, a B-box type 1, and a B-box type 2, plus a coiled-coil region, and six NHL domains. TRIM32 is widely expressed in numerous tissues ${ }^{36}$ and its importance in skeletal muscle homeostasis has already been established as point mutations were reported in autosomal recessive limbgirdle muscular dystrophy type 2H (LGMD2H) (MIM254110), first described in the Manitoba Hutterite population. ${ }^{37}$ So far, seven mutations in the TRIM32 gene have been linked to LGMD2H: six of them ${ }^{38-43}$ are clustered in the NHL domain at the C-terminal end and affect highly conserved residues. 

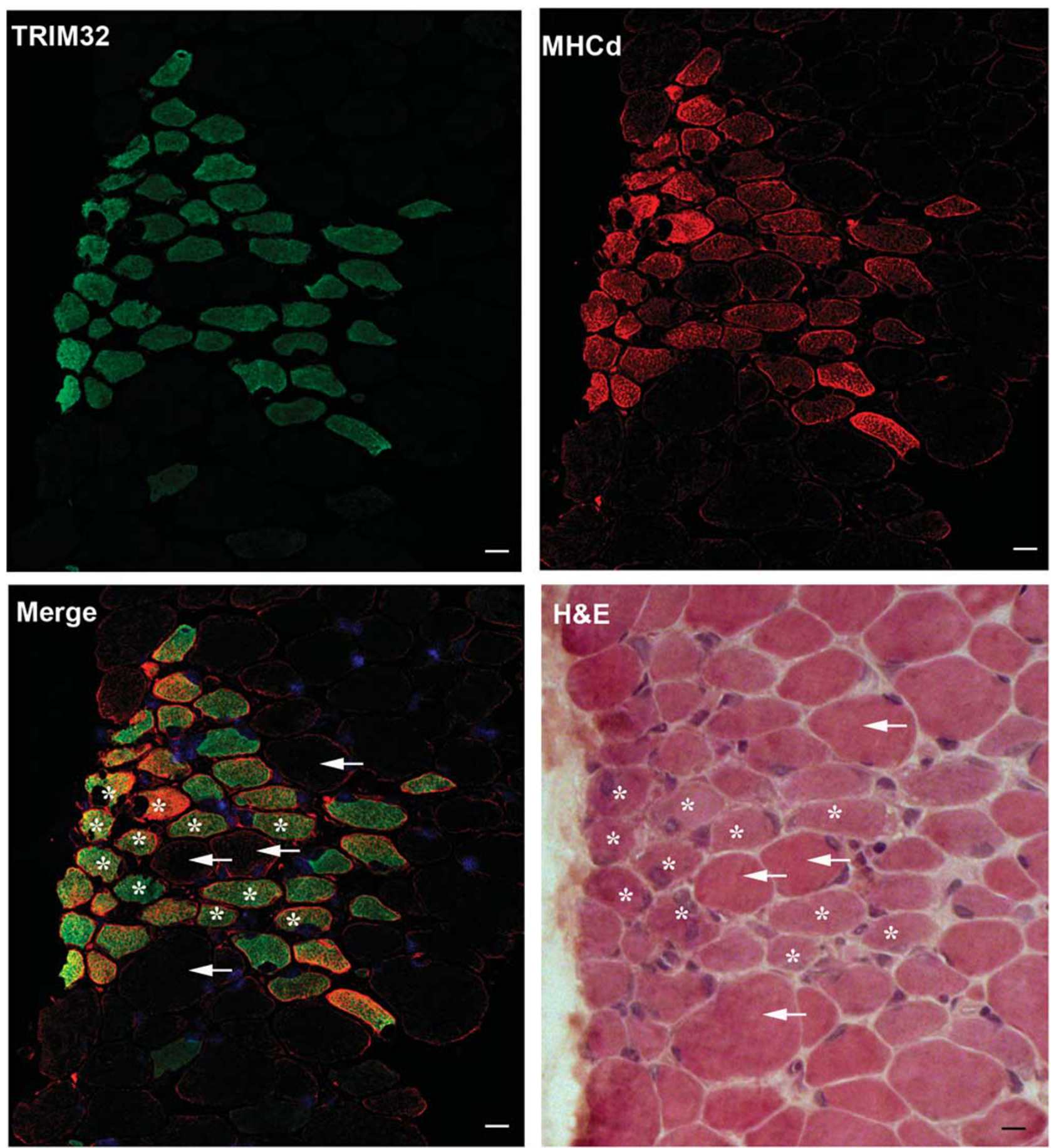

Figure 5 TRIM32 immunostaining in DMD muscle biopsies co-localized with the regeneration marker MHCd. Frozen skeletal muscle sections from BMD patients were immunostained with monoclonal antibodies directed against TRIM32 (green) and MHCd (red). Serial sections were stained with H\&E. Asterisks indicate basophilic TRIM32 and MHCd positive myofibers; white arrows indicate hypertrophic TRIM32 and MHCd negative cells. The images were acquired by confocal microscopy. Original magnification, $\times 40$. Scale bars $=20 \mu \mathrm{m}$. Representative images are shown.

In addition to LGMD2H, TRIM32 mutations can cause Bardet-Biedl syndrome, ${ }^{44}$ which is characterized by retinal degeneration, obesity, cognitive impairment, renal, and cardiovascular anomalies. Finally, selective up-regulation was reported in several types of cancer, ${ }^{45}$ in the occipital lobe of patients with Alzheimer's disease ${ }^{46}$, and in psoriatic lesions. ${ }^{47}$

In skeletal muscle, TRIM32 exerts pleiotropic effects, presumably depending on the distinct state of differentiation 
of muscle cells, i.e., adult myotubes $v s$ myogenic precursors. The Ub-ligase is involved in the ubiquitination of tissue-specific targets including myosin, $\operatorname{actin}^{48}$ and dysbindin. ${ }^{49}$ During muscle wasting, it catalyzes the loss of thin filaments and Z-band components through the regulation of the cytoskeletal protein, desmin, thus contributing to the disorganization of the cytoskeleton. ${ }^{50}$ TRIM32 may also influence muscle mass as its inhibition in normal myotubes enhances Phospho-Inositol-3Kinase (PI3K)-Akt-FoxO signaling by preventing the dissociation of plakoglobin from PI3K-p85. ${ }^{51}$

Surprisingly, TRIM32 knock-out mice display a myopathy, which has been mainly attributed to impaired muscle growth with satellite cell senescence and premature sarcopenia. ${ }^{52}$ In the two experimental models of muscle atrophy (fasting and disuse) and upon cardiotoxin-induced acute tissue damage, TRIM32 is selectively increased in satellite cells and regenerative/regrowth areas. Here, the Ub-ligase controls the turnover of at least three proteins, c-myc, N-myc downstream-regulated gene (NDRG2), and E3 SUMO ligase Piasy ${ }^{53-55}$, whose accumulation results in decreased myoblast proliferation and delayed-cell cycle withdrawal during myogenic differentiation. ${ }^{53,56}$

Our results demonstrated that, in human dystrophindeficient tissue, TRIM32 is selectively up-regulated in clusters of regenerative myofibers suggesting that the biological action of this Ub-ligase, in human dystrophic muscle, consists in a favourable induction of muscle growth rather than enhancement of muscle damage.

In this context, TRIM32 increase in DMD is consistent with the physiopathology of the disorder in which unrelenting, recurrent myofiber impairment elicits a constant need for regeneration. The rapid onset of muscle damage observed in DMD patients has been related, at least in part, to the depletion of functional muscle stem cells, which is the result of the continuous degeneration/regeneration cycling that occurs in their skeletal muscles due to plasma membrane instability and leakiness. Exhaustion of this regeneration potential ultimately leads to replacement of the skeletal muscle with adipose and fibrotic connective tissue. ${ }^{57}$

TRIM32-related mechanisms of action in satellite cells in DMD and possibly in other muscular dystrophies are particularly intriguing as there are multiple experimental strategies aimed at enhancing the regeneration of skeletal muscle in these disorders.

Moreover, the induction of a Ub-ligase in the pool of regenerative fibers further emphasizes the complexity of the regulation of intracellular protein homeostasis in pathological conditions. Future studies aimed at shaping proteostasis in DMD will have to consider that an excessive activation as well as a substantial inhibition of protein degradation, can concur to muscle degeneration by different mechanisms. ${ }^{5}$

\section{ACKNOWLEDGMENTS}

We would like to thank Dr Paolo Broda for expert technical advice. The financial support was given by G. Gaslini Foundation, the Ministero della Salute, Ricerca Corrente Del.74/13 to SA, EG; Association Francaise pour le Maladie Muscolaire, Ricerca Regionale Liguria to CM.

\section{DISCLOSURE/CONFLICT OF INTEREST}

The authors declare no conflict of interest.

1. Mercuri E, Muntoni F. Muscular dystrophy: new challenges and review of the current clinical trials. Curr Opin Pediatr 2013;25:701-707.

2. Kornegay JN, Spurney CF, Nghiem PP, et al. Pharmacologic management of Duchenne muscular dystrophy: target identification and preclinical trials. ILAR J 2014;55:119-149.

3. Assereto S, Stringara S, Sotgia F, et al. Pharmacological rescue of the dystrophin-glycoprotein complex in Duchenne and Becker skeletal muscle explants by proteasome inhibitor treatment. Am J Physiol Cell Physiol 2006;290:C577-C582.

4. Lilienbaum A. Relationship between the proteasomal system and autophagy. Int J Biochem Mol Biol 2013;4:1-26.

5. Sandri M, Coletto L, Grumati P, et al. Misregulation of autophagy and protein degradation systems in myopathies and muscular dystrophies. J Cell Sci 2013;126:5325-5333.

6. Chen CN, Graber TG, Bratten WM, et al. Immunoproteasome in animal models of Duchenne muscular dystrophy. J Muscle Res Cell Motil 2014:35:191-201.

7. Spitali $P$, Grumati $P$, Hiller $M$, et al. Autophagy is impaired in the tibialis anterior of dystrophin null mice. PLoS Curr 2013; 5-18.

8. Bonuccelli $G$, Sotgia F, Capozza $F$, et al. Localized treatment with a novel FDA-approved proteasome inhibitor blocks the degradation of dystrophin and dystrophin-associated proteins in mdx mice. Cell Cycle 2007;6:1242-1248.

9. Gazzerro E, Assereto S, Bonetto A, et al. Therapeutic potential of proteasome inhibition in Duchenne and Becker muscular dystrophies. Am J Pathol 2010;176:1863-1877.

10. Wadosky KM, Li L, Rodríguez JE, et al. Regulation of the calpain and ubiquitin-proteasome systems in a canine model of muscular dystrophy. Muscle Nerve 2011;44:553-562.

11. Araujo KP, Bonuccelli G, Duarte CN, et al. Bortezomib (PS-341) treatment decreases inflammation and partially rescues the expression of the dystrophin-glycoprotein complex in GRMD dogs. PLoS One 2013;8:e61367.

12. Maddocks K, Blum KA. Treatment strategies in mantle cell lymphoma. Cancer Treat Res. 2015;165:251-270.

13. Colson K. Treatment-related symptom management in patients with multiple myeloma: a review. Support Care Cancer 2015;23:1431-1445.

14. McBride A, Klaus JO, Stockerl-Goldstein K. Carfilzomib: a second-generation proteasome inhibitor for the treatment of multiple myeloma. Am J Health Syst Pharm 2015;72:353-360.

15. Selsby J, Morris $C$, Morris $L$, et al. A proteasome inhibitor fails to attenuate dystrophic pathology in mdx mice. PLoS Curr 2012;4:e4f84a944d8930.

16. Hollinger K, Selsby JT. The physiological response of protease inhibition in dystrophic muscle. Acta Physiol (Oxf) 2013;208:234-244.

17. Tomko RJ Jr, Hochstrasser M. Molecular architecture and assembly of the eukaryotic proteasome. Annu Rev Biochem 2013;82:415-445.

18. Finley D. Recognition and processing of ubiquitin-protein conjugates by the proteasome. Annu Rev Biochem 2009;78:477-513.

19. Bodine $S C$, Latres $E$, Baumhueter $S$, et al. Identification of ubiquitin ligases required for skeletal muscle atrophy. Science 2001;294:1704-1708.

20. Arndt V, Dick N, Tawo R, et al. Chaperone-assisted selective autophagy is essential for muscle maintenance. Curr Biol 2010;20:143-148.

21. Tocchini C, Ciosk R. TRIM-NHL proteins in development and disease. Semin Cell Dev Biol 2015:47-48:52-59.

22. Nagpal P, Plant PJ, Correa J, et al. The ubiquitin ligase nedd4-1 participates in denervation-induced skeletal muscle atrophy in mice. PLoS ONE 2012;7:e46427.

23. Nastasi $T$, Bongiovanni $A$, Campos $Y$, et al. Ozz-E3, a muscle-specific ubiquitin ligase, regulates beta-catenin degradation during myogenesis. Dev Cell 2004;6:269-282.

24. Vandesompele J, De Preter K, Pattyn F, et al. Accurate normalization of real-time quantitative RT-PCR data by geometric averaging of multiple internal control genes. Genome Biol 2002;3:RESEARCH0034. 
25. Hellemans J, Mortier G, De Paepe A, et al. qBase relative quantification framework and software for management and automated analysis of real-time quantitative PCR data. Genome Biol 2007;8:R19.

26. Briguet A, Erb M, Courdier-Fruh I, et al. Effect of calpain and proteasome inhibition on $\mathrm{Ca} 2+-$ dependent proteolysis and muscle histopathology in the mdx mouse. FASEB J 2008;22:4190-4200.

27. Gastaldello S, D'Angelo S, Franzoso S, et al. Inhibition of proteasome activity promotes the correct localization of disease-causing alphasarcoglycan mutants in HEK-293 cells constitutively expressing beta-, gamma-, and delta-sarcoglycan. Am J Pathol 2008;173:170-181.

28. Azakir BA, Erne B, Di Fulvio $S$, et al. Proteasome inhibitors increase missense mutated dysferlin in patients with muscular dystrophy. Sci Transl Med 2014;6:250ra112.

29. Fanin M, Nascimbeni AC, Angelini C. Muscle atrophy, ubiquitin-proteasome, and autophagic pathways in dysferlinopathy. Muscle Nerve 2014;50:340-347.

30. Rajakumar D, Senguttuvan S, Alexander $M$, et al. Involvement of oxidative stress, nuclear factor kappa $B$ and the ubiquitin proteasomal pathway in dysferlinopathy. Life Sci 2014;108:54-61.

31. Azakir BA, Di Fulvio S, Kinter J, et al. Proteasomal inhibition restores biological function of mis-sense mutated dysferlin in patient-derived muscle cells. J Biol Chem 2012;287:10344-10354.

32. Körner Z, Fontes-Oliveira CC, Holmberg J, et al. Bortezomib partially improves laminin a2 chain-deficient muscular dystrophy. Am J Pathol 2014;184:1518-1528.

33. Carmignac $V$, Quéré $R$, Durbeej $M$. Proteasome inhibition improves the muscle of laminin a2 chain-deficient mice. Hum Mol Genet 2011;20: 541-552.

34. Bianchini $E$, Fanin $M$, Mamchaoui $K$, et al. Unveiling the degradative route of the V247M a-sarcoglycan mutant responsible for LGMD-2D. Hum Mol Genet 2014;23:3746-3758.

35. Reymond A, Meroni G, Fantozzi $A$, et al. The tripartite motif family identifies cell compartments. EMBO J 2001;20:2140-2151.

36. Metzger MB, Hristova VA, Weissman AM. HECT and RING finger families of E3 ubiquitin ligases at a glance. J Cell Sci 2012;125:531-537.

37. Frosk $\mathrm{P}$, Weiler $\mathrm{T}$, Nylen $\mathrm{E}$, et al. Limb-girdle muscular dystrophy type $2 \mathrm{H}$ associated with mutation in TRIM32, a putative E3-ubiquitinligase gene. Am J Hum Genet 2002;70:663-672.

38. Frosk P, Del Bigio MR, Wrogemann $\mathrm{K}$, et al. Hutterite brothers both affected with two forms of limb girdle muscular dystrophy: LGMD2H and LGMD2I. Eur J Hum Genet 2005;13:978-982.

39. Saccone $V$, Palmieri $M$, Passamano $L$, et al. Mutations that impair interaction properties of TRIM32 associated with limb-girdle muscular dystrophy $2 \mathrm{H}$. Hum Mutat 2008;29:240-247.

40. Borg K, Stucka R, Locke M, et al. Intragenic deletion of TRIM32 in compound heterozygotes with sarcotubular myopathy/LGMD2H. Hum Mutat 2009;30:E831-E844.

41. Cossée M, Lagier-Tourenne $C$, Seguela $C$, et al. Use of SNP array analysis to identify a novel TRIM32 mutation in limb-girdle muscular dystrophy type 2H. Neuromuscul Disord 2009;19:255-260.
42. Liewluck T, Tracy JA, Sorenson EJ, et al. Scapuloperoneal muscular dystrophy phenotype due to TRIM32-sarcotubular myopathy in South Dakota Hutterite. Neuromuscul Disord 2013;23:133-138.

43. Neri $M$, Selvatici $R$, Scotton $C$, et al. A patient with limb girdle muscular dystrophy carries a TRIM32 deletion, detected by a novel CGH array, in compound heterozygosis with a nonsense mutation. Neuromuscul Disord 2013;23:478-482.

44. Chiang AP, Beck JS, Yen HJ, et al. Homozygosity mapping with SNP arrays identifies TRIM32, an E3 ubiquitin ligase, as a Bardet-Biedl syndrome gene (BBS11). Proc Natl Acad Sci U S A. 2006;103:6287-6292.

45. Horn EJ, Albor A, Liu Y, et al. RING protein Trim32 associated with skin carcinogenesis has anti-apoptotic and E3-ubiquitin ligase properties. Carcinogenesis 2004;25:157-167.

46. Yokota T, Mishra $M$, Akatsu $H$, et al. Brain site-specific gene expression analysis in Alzheimer's disease patients. Eur J Clin Invest 2006;36:820-830.

47. Liu Y, Lagowski JP, Gao S, et al. Regulation of the psoriatic chemokine CCL20 by E3 ligases Trim32 and Piasy in keratinocytes. J Invest Dermatol 2010;130:1384-1390

48. Kudryashova E, Kudryashov D, Kramerova I, et al. Trim32 is an ubiquitin ligase mutated in limb girdle muscular dystrophy type $2 \mathrm{H}$ that binds to skeletal muscle myosin and ubiquitinates actin. J Mol Biol 2005;354: 413-424.

49. Locke $\mathrm{M}$, Tinsley $\mathrm{CL}$, Benson $\mathrm{MA}$, et al. TRIM32 is an $\mathrm{E} 3$ ubiquitin ligase for dysbindin. Hum Mol Genet 2009;18:2344-2358.

50. Cohen S, Zhai B, Gygi SP, et al. Ubiquitylation by Trim32 causes coupled loss of desmin, Z-bands, and thin filaments in muscle atrophy. J Cell Biol 2012;198:575-589.

51. Cohen $\mathrm{S}$, Lee $\mathrm{D}$, Zhai $\mathrm{B}$, et al. Trim32 reduces PI3K-Akt-FoxO signaling in muscle atrophy by promoting plakoglobin-PI3K dissociation. J Cell Biol 2014:204:747-758.

52. Kudryashova E, Wu J, Havton LA, et al. Deficiency of the E3 ubiquitin ligase TRIM32 in mice leads to a myopathy with a neurogenic component. Hum Mol Genet 2009;18:1353-1367.

53. Albor A, El-Hizawi S, Horn EJ, et al. The interaction of Piasy with Trim32, an E3-ubiquitin ligase mutated in limb-girdle muscular dystrophy type $2 \mathrm{H}$, promotes Piasy degradation and regulates UVB-induced keratinocyte apoptosis through NFkappaB. J Biol Chem 2006;281: 25850-25866.

54. Nicklas $\mathrm{S}$, Otto $\mathrm{A}, \mathrm{Wu} \mathrm{X}$, et al. TRIM32 regulates skeletal muscle stem cell differentiation and is necessary for normal adult muscle regeneration. PLoS One 2012;7:e30445.

55. Kudryashova E, Kramerova I, Spencer MJ. Satellite cell senescence underlies myopathy in a mouse model of limb-girdle muscular dystrophy 2H. J Clin Invest 2012;122:1764-1776.

56. Mokhonova El, Avliyakulov NK, Kramerova I, et al. The E3 ubiquitin ligase TRIM32 regulates myoblast proliferation by controlling turnover of NDRG2. Hum Mol Genet 2015;24:2873-2883.

57. de Souza GT, Zanette Rde $\mathrm{S}$, do Amaral DL, et al. Satellite cells: regenerative mechanisms and applicability in muscular dystrophy. Stem Cells Int 2015; 487467. 